\title{
A multiobjective approach towards weapon assignment in a ground-based air defence environment
}

\author{
DP Lötter* \\ I Nieuwoudt ${ }^{\dagger}$ \\ JH van Vuuren \\ Received: 10 November 2012; Revised: 17 January 2013; Accepted: 27 January 2013

\section{Dedication to Emeritus Professor Theodor Stewart}

Dedicated with respect and appreciation to Theo Stewart, one of the forerunners in the international multi-criteria decision analysis (MCDA) arena, on the special occasion of his $70^{\text {th }}$ birthday. Not only has Theo made a large and significant contribution to the field of MCDA, but he also served the Operations Research Society of South Africa for many years in a number of capacities, amongst them as president of the Society and founding editor of ORiON. We celebrate his considerable impact on Operations Research, both locally and internationally, and especially on the development of MCDA theory and practice. Happy birthday Theo, may you be blessed with many more!

\begin{abstract}
A typical ground-based air defence (GBAD) environment comprises defended assets on the ground which require protection from enemy aircraft entering the defended airspace. Protection against these aircraft is afforded by means of pre-deployed ground-based weapon systems that are assigned to engage these enemy aircraft according to some pre-specified criterion or set of criteria. The conditions under which human operators have to propose assignments of weapon systems to engage these aircraft are severely stressful since time is a critical factor and there is no room for error. Some progress has already been made with respect to the design of computerised threat evaluation and weapon assignment (TEWA) decision support systems (DSSs) within the context of a GBAD system. However, the weapon assignment (WA) component within such a TEWA DSS is typically based on a single criterion (objective). The aim in this paper is to model the WA problem as a multiobjective decision problem. A list of relevant factors (related to objectives) is identified by means of feedback received from a WA questionnaire which was completed by a number of military experts. For illustrative purposes, two objectives, namely the cost of assigning weapon systems for engagement and the accumulated survival probabilities of observed threats as a result of these engagements, were isolated from these factors in order to derive a bi-objective WA model. This model is solved in the context of a simulated, but realistic, GBAD environment by means of an existing multiobjective solution technique called the Nondominated Sorting Genetic Algorithm II.
\end{abstract}

Key words: Multicriteria decision making, threat evaluation and weapon assignment, NSGA II.

\footnotetext{
${ }^{*}$ Corresponding author: Department of Logistics, University of Stellenbosch, Private Bag X1, Matieland, 7602, South Africa, email: 14556561@sun.ac.za

${ }^{\dagger}$ Department of Logistics, University of Stellenbosch, Private Bag X1, Matieland, 7602, South Africa.

$\ddagger$ (Fellow of the Operations Research Society of South Africa), Departments of Logistics, University of Stellenbosch, Private Bag X1, Matieland, 7602, South Africa.
} 


\section{Introduction}

In a typical Ground-Based Air Defence (GBAD) environment, Defended Assets (DAs) on the ground require protection from enemy aircraft entering the volume of space surrounding the DAs, known as the defended airspace. Such protection is usually afforded by means of pre-deployed ground-based Weapon Systems (WSs). A network of sensors is typically responsible for detecting aircraft entering the defended airspace. Once aircraft have been detected, they are labelled and classified according to the perceived level of threat they pose to the DAs. Upon this classification of threats, WSs have to be assigned to engage these threats. A Fire Control Officer (FCO) is responsible for assigning ground-based WSs to engage these threats. This assignment problem is known as the Weapon Assignment (WA) problem.

The FCO has to make assignment decisions under severely stressful conditions, since the speed at which aerial threats approach the DAs leaves little time for analysis or delays in reaction time. Not only does the FCO have to decide which WSs to assign to the aerial threats, but he also has to choose the number of WSs to assign to each individual threat. The assignment of multiple WSs to a threat may result in an increased probability of eliminating that specific threat, but may simultaneously compromise the number of WSs available for assignment during future time intervals, called stages.

Apart from deciding which WSs to assign to a specific threat, the FCO also has to decide when these engagements should take place (i.e. whether he should assign any WS during the current stage, or whether he should rather wait until a later stage when the probability of eliminating the threat is higher). Moreover, a WS achieving a longer range typically involves a higher monetary cost of assignment than does a WS achieving a shorter range.

When a large number of aircraft approach the defended airspace almost simultaneously from various directions, the WA problem becomes very complex and almost impossible for the FCO to solve close to optimally in real-time. For this reason, a computerised Threat Evaluation and Weapon Assignment (TEWA) Decision Support System (DSS) is often employed to provide the FCO with one or more high-quality alternatives (possible assignments). Using his own judgment, which is typically based on considerable experience and training, in conjunction with the alternatives recommended by the TEWA DSS, he is expected to be able to make more effective assignment decisions with respect to the engagement of aerial threats by WSs, and to make these assignment decisions with more confidence.

Such a TEWA DSS usually consists of two separate subsystems called a Threat Evaluation (TE) subsystem and a WA subsystem. The TE subsystem is responsible for evaluating the level of threat that enemy aircraft pose to the DAs, whilst the WA subsystem is responsible for providing high-quality assignment proposals of WSs to engage the aerial threats.

Assignment decisions are proposed by the WA subsystem based on the solution of one or more WA models. Typical WA models found in the literature involve only single objective optimisation, where the aim is usually to maximise the overall or accumulated probability of successful engagement of aerial threats by WSs. This overall probability of successful engagement is quantified by means of so-called Single Shot Hit Probability (SSHP) information related to the WSs deployed. The aim in this paper is, however, 
to model the WA problem as a multiobjective decision problem, and to solve it in the context of a simulated, but realistic, GBAD scenario as a proof of concept, demonstrating the workability of the newly proposed multiobjective decision model. This requires the identification of a number of fundamental objectives deemed relevant by South African military experts and incorporating some subset of these objectives into a multiobjective decision framework.

This paper is organised in the following manner. The working of a typical TEWA DSS as part of a GBAD environment is briefly described in $\S 2$, with the emphasis on TE in $\S 2.1$ and WA in $§ 2.2$. Various well-known single objective WA models from the literature are briefly reviewed in $\S 3$. This is followed, in $\S 4$, by a discussion on the procedures followed by the authors to construct a bi-objective WA model, as well as a brief discussion on the working of the well-known Nondominated Sorting Genetic Algorithm II (NSGA II) for solving multicriterion optimisation problems in a population-based manner. A simulated, but realistic, GBAD scenario is described in $\S 5.1$ and an implementation of our proposed bi-objective WA model and the results thereof in the context of this scenario are discussed in $\S 5.2$ and $\S 5.3$, respectively. The paper closes, in $\S 6$, with a number of suggestions with respect to future work related to the multiobjective WA problem.

\section{TEWA in a GBAD environment}

Upon detection of aerial vehicles entering the defended airspace by a network of remote sensors, these vehicles are typically labelled and classified according to their hostility category $^{1}$ and platform type ${ }^{2}$ by means of two processes contained in a so-called Track Management (TM) process. Hostility classification is achieved by means of a process called Hostility Classification/Identification (HCI) and usually involves the classification of aircraft as either hostile, friendly or unknown. The identification of the platform type of aerial vehicles is achieved by means of a process called Type Classification/ Identification (TCI) and involves the classification of aircraft into different platform types such as rotary wing, fixed wing, cargo, missile, unmanned aerial vehicle, electronic warfare platform or unknown [17]. The TM is also responsible for creating a so-called system track for each of the detected aircraft by means of a fusing process based on information from the sensors, such as the speed, altitude and direction of the aircraft.

\subsection{The TE subsystem}

The TE subsystem only considers system tracks of aerial vehicles which have been classified as hostile or unknown. The system tracks of these aerial threats are analysed in real-time by the TE subsystem according to their capability and intent. The capability of a threat is a reflection of its ability to inflict injury or damage to the DAs. Factors which influence the capability of a threat include the proximity of the threat to the DAs and the characteristics of weapons carried by the threat. The intent of a threat refers to the will or determination

\footnotetext{
${ }^{1}$ The hostility of an aircraft refers to its ability and intent to inflict damage to DAs, based on some pre-specified set of criteria, and may be catergorised into different classes.

${ }^{2}$ Aircraft may be divided into different classes of platform types, where a specific platform type encompasses aircraft involving the same characteristics, such as, for example, fixed wing aircraft.
} 
of the threat to reach the various DAs in order to inflict damage or injury to them [17]. Factors which may be used to ascertain the intent of a threat include the velocity of the threat, its course and altitude, and an estimation of the technique adopted by the enemy pilot in order to deliver the weapons carried by his aircraft to one or more DAs.

The capability of a threat is usually easier to estimate than its intent. It is important that a TE subsystem is sophisticated enough to explore all the available information with respect to an aircraft which enters the defended airspace and at the same time be capable of producing a result based on scant information [13]. A solution to this problem was proposed by Roux and Van Vuuren [18], who suggested the use of a suite of TE models, consisting of qualitative and quantitative models, which function concurrently. The qualitative models are binary models which are able to flag human operators in the event of a change in the kinematic behaviour of enemy aircraft, whereas the quantitative models are able to distinguish between different levels of threat posed by the aircraft. The output of each quantitative TE model is a prioritised list of the observed threats with respect to each DA, based on a so-called threat value allocated to each threat. This value is an indication on a suitable scale of threat values of the level of threat that it poses to the DA. The results from the various TE models may then be fused together to obtain a single threat list with respect to all DAs.

The suite of TE models proposed in [18] encompasses the following three classes of models in order of increasing sophistication:

Flagging models (qualitative). These models are only concerned with reporting sudden changes in aircraft behaviour, and they function on the basis that if any attribute of the detected aircraft deviates significantly from that of their current or past values, a human operator is flagged.

Deterministic models (quantitative). These models use measures, such as the distance between the aircraft and the DAs or the aircraft's bearing with respect to DAs, to generate threat values and threat lists.

Stochastic models (quantitative). These models attempt to make use of probability values, such as the probability of attack or the probability of kill, to express the threat that aircraft pose to the respective DAs.

\subsection{The WA subsystem}

The WA subsystem suggests an assignment of available WSs to threats in order to achieve a pre-specified objective. The extent to which the objective is achieved may be seen as the desirability of the specific WS-to-threat assignments. Examples of such objectives include minimising the expected aggregated damage to DAs, maximising the number of threats engaged or maximising the expected aggregated damage to threats [13].

The WA subsystem requires the output (i.e. a prioritised GBAD system threat list) provided by the TE subsystem in conjunction with an array of WS-related data, called an Engagement Efficieny Matrix (EEM), as input. The threat list and the EEM are presented to the WA subsystem in real-time and updated by the TE subsystem as new kinematic 
information related to the system tracks of the aerial threats become available. The EEM is a three-dimensional matrix, containing the predicted engagement efficiency values of the WSs with respect to each of the observed threats; its dimensions are WSs deployed, threats and time. This matrix is computed by using SSHP information, filtered by other information such as weather and terrain feature data [13]. SSHP values are normally supplied by the manufacturers of WSs and are based on the characteristics of the specific type of WS. The output of the WA subsystem is a proposed assignment list of WSs to aerial threats in the form of WS-threat pairings.

\section{A brief review of WA models from the literature}

The WA problem has its roots in the Classical Assignment Problem (CAP), which was introduced by Votaw and Orden [21] in 1952. The CAP deals with the problem of assigning $n$ agents to $n$ tasks so as to minimise the overall cost of the assignments. The CAP was used as a stepping stone by Flood [7], in 1957, who introduced the Weapon Target Assignment (WTA) problem, and by Manne [12], who later in that same year formulated the WTA as an integer programming problem. The formulation of the WTA problem by Manne is a nonlinear modification of the CAP, where the agents are replaced by $m$ uniform WSs and the tasks are replaced by $n$ threats. The objective in the WTA problem is to determine those WS-threat engagement pairs that minimise the overall expected survivability of the threats. Assumptions typically made are that the SSHP of a WS with respect to a threat depends only on the WS and threat involved, and that the events of a threat surviving engagements by two different WSs are independent.

Since the original formulation of the WTA in 1957, a number of authors have contributed generalisations to the WTA. In 2003, for example, Ahuja et al. [2] slightly adjusted the formulation of the WTA problem by introducing different WS types in the formulation. Another generalisation is that the objective in the WTA problem by Ahuja et al. is to minimise the accumulated weighted probability of survival of the observed threats. The probability that a threat will survive is calculated as the product of the probabilities of surviving separate WS engagements assigned to it. This probability of survival is then weighted by the priorities of eliminating the threats in the threat list.

The WTA problem was further adjusted by Potgieter [13] in 2008 to make provision for the assignment of at most $k$ WSs to a single threat. This model is constrained in two ways, namely by limiting each WS to be assigned at most once, and also limiting the number of WSs assigned to any threat to at most $k$.

Although other variations of the WTA problem may be found in the literature, these variations are all limited to a single objective, usually minimising overall survivability of the aerial threats.

\section{Model design}

A first step in the modelling process of a multiobjective decision problem is to identify the desired objectives of the decision maker. This identification process usually involves a 
decision analyst or a facilitator interviewing the decision maker or, in some cases, group of decision makers. During such interviews, the decision maker(s) may be asked a series of questions which may lead the decision analyst to a number of desirable objectives. The decision analyst may further probe the decision maker(s) about their initial responses in order to induce them to think in more depth about their initial responses so as to ensure that all aspects of the problem are covered. Keeney [9] proposes a number of techniques and questions which may be employed during such interviews.

When the insights of a number of decision makers are sought, a series of group facilitated workshops may also be conducted to identify a comprehensive set of fundamental objectives. These workshops may vary in their degree of intensity. A workshop involving a simple discussion between the decision analyst and team of decision makers may be sufficient to capture the necessary information in the context of an uncomplicated decision. However, in the case where a decision problem involves a higher degree of complexity, more comprehensive infusion procedures may be required to capture a more detailed set of objectives [3].

Belton and Stewart [3] suggest two useful methods which may be employed during facilitated workshops, known as the Delphi method and Post-it ${ }^{3}$ sessions:

1. A post-it session involves the distribution of post-it notepads amongst the group of decision makers. They are required to express their ideas and the facts which they deem important on the notepads. Group members are then invited to stick their notes to a flat surface, which is visible to all team members, in order for their ideas to be shared and discussed amongst each other, possibly generating further ideas.

2. The Delphi method, on the other hand, aims to structure the communication process in a group facilitated context [10]. The conventional Delphi method entails a team of respondents (usually experts in the relevant field) who are required to complete a questionnaire, concerning relevant questions related to the problem at hand [8]. The questionnaire is usually developed prior to the workshop and handed to the decision makers to complete. Once the questionnaires have been completed the results are summarised and analysed by the workshop facilitator. The results are then presented anonymously to the group of decision makers and they are given an opportunity to review the responses of the entire group, based on the results obtained in the first round [10]. The process may be repeated until a stopping criterion is satisfied, such as reaching a maximum number of rounds or until consensus is reached.

Identifying model objectives may be a painstaking process, but a well-structured decision model may significantly simplify the decision making process at a later stage.

\subsection{Identification of WA objectives}

It is difficult to isolate a single individual who is able to act as the sole decision maker in the matter of WA decisions; it seems more desirable to identify a number of experts, residing within the South African military domain, who collectively act as the decision

\footnotetext{
${ }^{3}$ Post-It refers to an item of stationary, called a post-it notepad, containing an adhesive strip on the back, allowing one to attach it to a surface [6].
} 
maker. Due to the small number of GBAD WA experts within the South African National Defence Force and the sensitive nature of the decision process at hand, a military expert (Visser [20]) was approached to aid in the identification of a further number of suitable military experts.

An ideal approach towards obtaining fundamental objectives with respect to WA decisions is to host a facilitated workshop with the group of military experts identified above during which interaction between the members of the group may lead to various ideas related to and different perspectives on the WA problem. However, due to limitations posed by the geographic dispersal of these individuals, hosting such an interactive workshop was not a viable option in the research project reported here. The closest alternative was to extract suitable WA decision objectives from responses to an electronic survey or questionnaire, which required separate completion by each individual in the group of military experts.

After consulting with TEWA DSS experts Potgieter [14] and Roux [15], as well as military expert Visser [20], a survey consisting of a set of key questions relating to various possible factors which may influence the choice of WS assignments in a GBAD context, was devised for this purpose. The majority of these questions were based on a specific GBAD scenario within the South African military context. In this way it was anticipated that the military experts who were required to complete the survey would relate more easily to the questions, especially if they were presented in conjunction with graphical illustrations. Since the military experts are typically trained to think about and analyse tactical situations in a specific scenario-based context, it was anticipated that they might find questions linked to a scenario context more compelling.

The majority of the scenario-based questions required the military experts to execute a choice between three possible WSs to assign to various approaching threats, located at various distances from the WSs during successive temporal stages of the scenario. They also had the opportunity not to assign any WSs during any given stage, implying that the threat should be engaged during a later stage. Each question related to the choice between WSs was followed by an opportunity during which the military experts could explain their decisions. These explanatory notes formed part of the core purpose of the survey, since this was where valuable information was gleaned. The reasoning behind the various choices invariably led to the identification of further factors which may influence the choice of WS assignments. An abridged version of the survey may be found in the Appendix at the end of the paper.

Feedback was received from the various military experts via military expert Visser [20]

\begin{tabular}{ll}
\hline Ability to engage behind obstacles & Night fight/fire capability \\
All weather capability & Reaction times of WSs \\
Availability of ammunition & Speed of WS ammunition \\
Cost of ammunition & SSHP information \\
Effective ranges of WSs & Terrain features \\
Line of sight information & Vertical launch capability \\
Multiple engageability & Weather conditions \\
\hline
\end{tabular}

Table 1: A list of factors which may influence the choice of assignment during the WA process, obtained from responses to the WA survey in the appendix at the end of this paper. 
who ensured anonymity of the respondents and that no classified information was included in the responses. A total of fourteen factors influencing WA assignment decisions were identified upon examination of the survey responses, as shown in Table 1.

Four fundamental objectives were derived as a result of the eleven factors in Table 1. These four objectives, together with the factors used to derive each of these objectives, are listed in Table 2. It was decided to include, as a proof of concept, the first two objectives in Table 2 in the derivation of our WA model. Choosing to incorporate only two objectives in our model also facilitates effective presentation of approximate fronts of Pareto optimal solutions in the form two-dimensional graphics.

\begin{tabular}{cll}
\hline No & Objective & Relevant factors from Table 1 \\
\hline 1 & Minimise aggregated survivability of & SSHP information \\
& observed aerial threats as a & Terrain features \\
& result of assignment & Line-of-sight information \\
& & Ability to engage behind obstacles \\
& & Weather conditions \\
& & All weather capability \\
& & Effective ranges of WSs \\
& & Vertical launch capability \\
\hline 2 & Minimise aggregated cost of assignment & Cost of ammunition \\
\hline 3 & Minimise maximum engagement time in & Speed of WS ammunition \\
& the assignment & \\
\hline 4 & Maximise minimum number of times a WS & Multiple engagement ability of WSs \\
& can re-engage after the assignment & Ammunition available for WSs \\
\hline
\end{tabular}

Table 2: WA objectives derived from the list of factors in Table 1.

\subsection{Formulation of a bi-objective WA model}

A bi-objective WA model, incorporating the first two objectives in Table 2 , is derived in this section. The first objective aims to maximise the overall assignment efficiency by using the SSHP values of WSs (which is equivalent to minimising the overall survivability of the aerial threats as a result of the WS assignment), whereas the second objective aims to minimise the overall cost of WSs assigned, as measured by the cost of ammunition in South African Rands. These objectives are conflicting in the sense that assigning more WSs to a threat, results in a higher overall expected assignment efficiency, but this will, in turn, result in a higher overall assignment cost. Hence, the aim is to find an acceptable trade-off between the cost and efficiency of WSs assigned.

Define the binary decision variable $x_{i j}$ to take the value 1 if WS $i$ is assigned to engage threat $j$, or the value 0 otherwise. Furthermore, let $q_{i j}$ be the probability that threat $j$ will survive a single shot aimed at it by WS $i$. As mentioned before, $q_{i j}$ is therefore the complement of the SSHP of WS $i$ when engaging threat $j$. Assuming that the separate events of survival of threat $i$ when engaged by multiple WSs are independent, the probability that threat $i$ will survive the engagements of all WSs assigned to it is given by the product $\prod_{i=1}^{n_{w}} q_{i j}^{x_{i j}}$, where $n_{w}$ denotes the number of WSs available for assignment. Denote the priority of eliminating threat $j$ by $B_{j}$ (this priority is typically the threat value 
assigned to threat $j$ by the TE subsystem) and denote the ammunition cost associated with a single shot of WS $i$ by $D_{i}$. Then the objectives of our bi-objective model are to

$$
\begin{aligned}
& \operatorname{minimise} \sum_{j=1}^{n_{t}} B_{j} \prod_{i=1}^{n_{w}} q_{i j}^{x_{i j}} \text {, } \\
& \operatorname{minimise} \sum_{i=1}^{n_{w}} D_{i} \sum_{j=1}^{n_{t}} x_{i j} \text {, }
\end{aligned}
$$

subject to the constraints

$$
\begin{array}{ll}
\sum_{j=1}^{n_{t}} x_{i j} \leq 1, & i=1, \ldots, n_{w} \\
x_{i j} \in\{0,1\}, & i=1, \ldots, n_{w} \\
& j=1, \ldots, n_{t},
\end{array}
$$

where $n_{t}$ denotes the number of threats observed. The objective in (1) is the same as in the adjusted WA model by Ahuja et al. [2], as discussed briefly in $\S 3$, while the objective in (2) is new and minimises the overall cost of the entire WS engagement with respect to all threats. The formulation is constrained by the assumption that a WS may only be assigned to one threat (multiple simultaneous engagements by a single WS is deemed impossible). However, the number of WSs assigned to any threat is limited only by the total number of WSs available, $n_{w}$. Finally, since the model is only valid for a single temporal stage (or engagement time-interval), the assumption is made that the various stages in the planning window under consideration are independent of one another, and that WSs are only considered for assignment during a single stage ${ }^{4}$. The implication of this assumption is that the efficiency of WAs during the current stage are not affected by assignments made during previous stages nor does the current assignment affect future assignment efficiencies. Each WS is therefore assumed to be available for assignment during each stage, while the threats present during any stage are assumed to be those that survived WS engagements during previous stages.

\subsection{Solving the model}

A number of multiobjective optimisation techniques exist in the literature which may be employed to solve the model in $\S 4.2$. These techniques typically focus on finding a set of Pareto optimal solutions (also called the nondominated set of solutions), that is a subset of solutions in the feasible region (3)-(4) which are superior to the remaining solutions in the feasible region (3)-(4) with respect to all the objectives, but are inferior to other solutions in the Pareto optimal set with respect to one or more objectives $[1,5]$.

An intuitive approach towards multiobjective problems is evolutionary algorithms. In these algorithms a pool of candidate solutions to an optimisation problem are allowed to

\footnotetext{
${ }^{4}$ It should be noted that although this assumption is appropriate in the context of the single time-period WA model (1)-(4), it is unrealistic in practice since the WA planning period typically spans a number of time steps.
} 
evolve in a carefully controlled manner over a number of iterations in the hope that they will eventually approximate the entire set of Pareto optimal solutions closely.

In this paper, a well-known multiobjective evolutionary algorithm, known as the Nondominated Sorting Genetic Algorithtm II [1], is employed to solve the bi-objective WA model (1)-(4). The NSGA II works in the following way. An initial population of candidate solutions of size $N$ is generated randomly. These solutions are then ranked and sorted by using the Fast Nondominated Sorting Algorithm (FNSA) [1] which has a computational complexity of $O\left(M N^{2}\right)$, where $M$ denotes the number of objectives in the multiobjective optimisation problem. For each solution $\boldsymbol{i}$, a dominance count $d_{\boldsymbol{i}}^{c}$ (the number of solutions that dominate $\boldsymbol{i}$ ), and a set of solutions $S_{\boldsymbol{i}}$ dominated by $\boldsymbol{i}$, are computed. All solutions achieving a dominance count value of $d_{\boldsymbol{i}}^{c}=0$ are placed in a separate set $\mathcal{F}_{1}$, called the first nondominated front, and are assigned rank 1 . For each solution $\boldsymbol{i}$ in $\mathcal{F}_{1}$, the algorithm cycles through each solution $\boldsymbol{j}$ in $S_{\boldsymbol{i}}$, and decrements its $d_{j}^{c}$ value by one, thus discounting the effect of solution $\boldsymbol{i}$ on solution $\boldsymbol{j}$ 's dominance count. All the rank 2 solutions now achieve a dominance count value of $d_{i}^{c}=0$, and are placed in a separate set $\mathcal{F}_{2}$ for the algorithm to cycle through. The algorithm continues in this fashion until all the solutions have been partitioned into ranks.

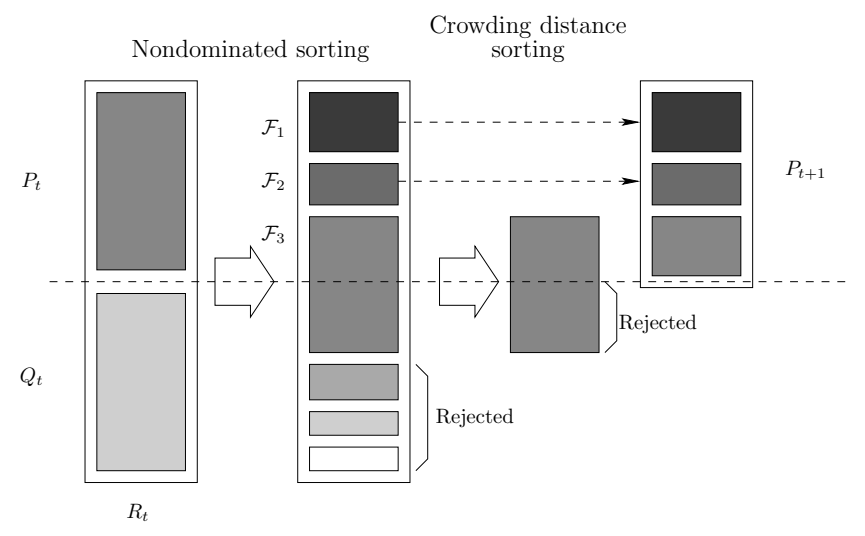

Figure 1: The crossover procedure of the NSGA II [4].

Next, a crowding distance density measure is calculated for each candidate solution in the population. This measure quantifies the density of solutions in the objective space and requires the solutions in the population to be sorted in ascending order of magnitude along each objective axis. Denote the objective function value of the $i^{\text {th }}$ candidate solution for the $h^{t h}$ objective (in the sorted list) by $X[i] \mid h$. The crowding distance for the $i^{t h}$ solution of the $h^{\text {th }}$ objective is then denoted by $i_{\text {dist }} \mid h$. An infinite crowding distance is assigned to the boundary solutions, that is the solutions $X[1] \mid h$ and $X[k] \mid h$, so as to ensure that they are selected for crossover. The crowding distances of the intermediate solutions $\boldsymbol{i}$ are incremented by the normalised distance between their closest neighboring solutions. More specifically, the normalised distance value is calculated as $(X[i+1] \mid h-$ $X[i-1] \mid h) /\left(h_{\max }-h_{\min }\right)$, where $h_{\max }$ and $h_{\min }$ represent the maximum and minimum values of the $h^{\text {th }}$ objective function, respectively. The overall crowding distance for each objective is taken as the accumulated value of the crowding distances of the individual solutions. The crowding distances are used to quantify the solution density in the sense 
that a higher value indicates that a solution is more isolated, while a lower value implies that a solution is more crowded by other solutions.

A crowding distance operator, denoted by $\prec_{c}$, is employed during the selection process of the algorithm. The rank assigned to a solution serves as the first criterion to determine superiority between solutions. A solution achieving a lower rank value is considered the superior solution, i.e. if $\mathcal{F}_{\boldsymbol{i}}<\mathcal{F}_{\boldsymbol{j}}$, then $\boldsymbol{i} \prec_{c} \boldsymbol{j}$ (denoting that solution $\boldsymbol{i}$ is superior to solution $\boldsymbol{j}$ ). However, if a pair of solutions achieves the same rank value, then the solution with the highest crowding distance is considered the superior solution as a means to break the tie, i.e. if $\mathcal{F}_{\boldsymbol{i}}=\mathcal{F}_{\boldsymbol{j}}$ and $\boldsymbol{i}_{\text {dist }}>\boldsymbol{j}_{\text {dist }}$, then $\boldsymbol{i} \prec_{c} \boldsymbol{j}$. In this way, more solutions are explored in less crowded regions of the solution space, which leads to a more uniformly spaced Pareto optimal frontier approximation.

The population of candidate solutions $\boldsymbol{P}_{t}$ during iteration $t$ of the NSGA II is sorted and ranked into fronts by applying the FNSA, as described above. Crowding distances are computed for each solution in $\boldsymbol{P}_{t}$ and an intermediate population $\boldsymbol{Q}_{t}$ of offspring is generated by performing binary tournament selection on the solutions $\boldsymbol{P}_{t}$. The selection is based on the crowding distance operator $\prec_{c}$, and the crossover and mutation operators are applied to the selected solutions. The parent and child solutions are combined to form a larger intermediate population $\boldsymbol{R}_{t}=\boldsymbol{P}_{t} \cup \boldsymbol{Q}_{t}$ of size $2 N$. This larger population $\boldsymbol{R}_{t}$ is then sorted and ranked into nondominated fronts by again applying the FNSA. The next population of candidate solutions $\boldsymbol{P}_{t+1}$ is generated by taking the solutions in the first front $\mathcal{F}_{1}$, then the solutions in the second front $\mathcal{F}_{2}$, and so forth, until the population size $N$ is reached. If all the solutions in a particular front cannot be included in $\boldsymbol{P}_{t+1}$, the solutions in that front are sorted in descending order with respect to their crowding distance and solutions are added to $\boldsymbol{P}_{t+1}$ in this order until a population size of $N$ is reached. An initial solution $\boldsymbol{P}_{0}$ is generated randomly and the algorithm is iterated until the generation counter $t$ reaches a prespecified maximum value. A graphical illustration of the crossover operation of the NSGA II may be found in Figure 1.

\section{A case study}

This section is devoted to an illustration of the working of the bi-objective WA model developed in $\S 4$. It is solved in the context of a simulated, but realistic GBAD scenario for three stages in the time continuum by means of the NSGA II. This section contains, in $§ 5.1$, a detailed description of the GBAD test scenario, in $\S 5.2$, a short discussion on the choice of the parameter values for the NSGA II and, in $\S 5.3$, a presentation and analysis of the numerical results obtained. The section closes with a discussion, in $§ 5.4$, on the advantages of solving the bi-objective WA model by means of a population-based algorithm.

\subsection{A realistic GBAD scenario}

The working of the bi-objective WA model (1)-(4) is illustrated by solving it in the context of a simulated GBAD scenario designed by Roux [16]. The scenario mimics a typical GBADS deployment in which twelve ground-based WSs provide protection to two DAs. 
A top view of the deployment is shown in Figure 2. The DAs are represented by black squares in the figure, labelled $\mathrm{DA}_{1}$ and $\mathrm{DA}_{2}$. The twelve WSs are deployed symmetrically in two concentric circles around the DAs and consist of eight Very SHOrt-Range Air Defence systems (VSHORADs) labelled $V_{1}, \ldots, V_{8}$ and four Close-In Weapon Systems (CIWSs) labelled $C_{1}, C_{2}, C_{3}, C_{4}$.

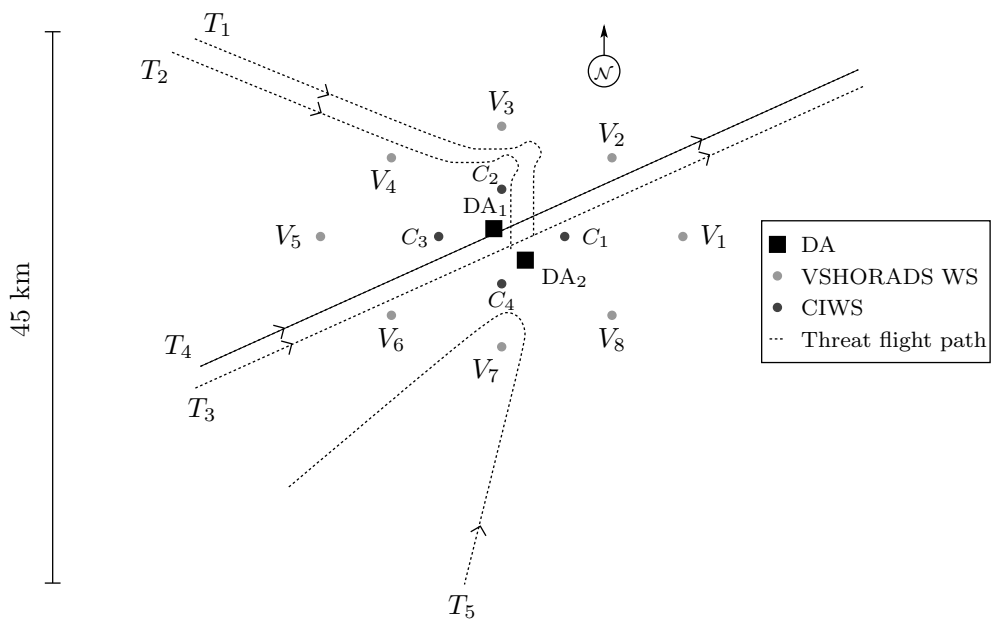

Figure 2: Top view of the simulated GBAD scenario.

In the scenario, five aircraft (labelled $T_{1}, T_{2}, T_{3}, T_{4}, T_{5}$ ) enter the defended airspace and are considered threats to the DAs. These aircraft approach the deployment area in three groupings, offset at different stages. The first group consists of threats $T_{1}$ and $T_{2}$ which enter the system from the north-west to attack $\mathrm{DA}_{2}$ according to a so-called pitch-anddive flight path attack technique. They fly in along a straight line, approaching at low altitude in an attempt at avoiding radar detection and initially their flight paths do not intersect the locations of the DAs, until they are at a distance of approximately $7500 \mathrm{~m}$ from $\mathrm{DA}_{1}$. Both of them pitch and turn in to $\mathrm{DA}_{2}$ at this point to dive straight onto $\mathrm{DA}_{2}$, releasing their weapons at an approximate distance of $800 \mathrm{~m}$ from $\mathrm{DA}_{2}$ [13]. The second group consists of threats $T_{3}$ and $T_{4}$ which merely act as decoys to the system. They enter the deployment area from the south-west and fly exactly over the DAs at high altitude $(4400 \mathrm{~m})$, whilst maintaining a constant speed of approximately $250 \mathrm{~ms}^{-1}$ until they exit towards the north-east, not attacking the DAs. Finally, threat $T_{5}$ enters from the South and attacks $\mathrm{DA}_{2}$ by means of a so-called toss bomb flight path attack technique. It enters the system in a straight line which does not intersect the locations of the DAs. It turns in towards $\mathrm{DA}_{2}$ (to the left) at an approximate distance of $9000 \mathrm{~m}$ from $\mathrm{DA}_{2}$ to release a bomb approximately $8000 \mathrm{~m}$ from the DA, after which it turns away and exits the system in a south-westerly direction [13].

The time continuum of the scenario is subdivided into 30 time intervals or stages. Each one of these stages spans a time interval of four seconds. The reason for this discretization is that radars employed for sensing are assumed to refresh the air picture every four seconds, updating the current positions of the threats. Since the bi-objective WA model (1)-(4) considers only a single stage at a time, and the stages are considered to be independent of one another, it was decided to select only a small number of stages for which to present 
solutions of the model, rather than showing results obtained from the model at each stage over the entire time continuum. The selected stages are $t_{20}, t_{35}$ and $t_{39}$. These stages were chosen for their diversity, and the locations of the threats during stages $t_{20}, t_{35}$ and $t_{39}$ are represented by the light grey, dark grey and black triangles, respectively, in Figure 3.

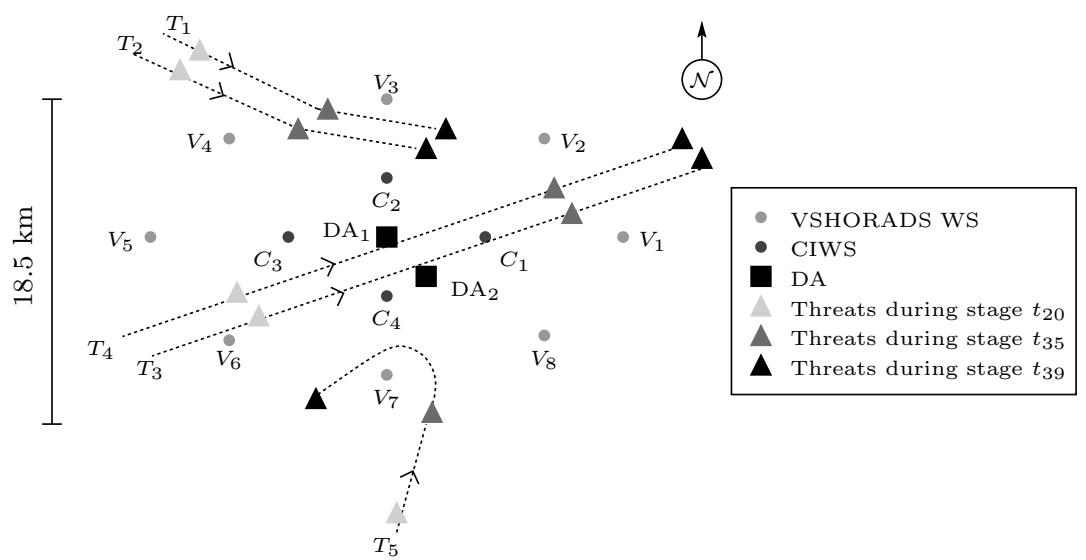

Figure 3: Top view of the locations of threats $T_{1}, T_{2}, T_{3}, T_{4}$ and $T_{5}$ in the simulated GBAD scenario in Figure 2 during stages $t_{20}, t_{35}$ and $t_{39}$.

Since the focus of this paper is on the WA subsystem within a larger TEWA system, as described in $\S 2$, it is assumed for simplicity that the TE process related to the scenario described above has been completed and that threat values have been assigned to each of the five threats for each of the three stages, as shown in Table 3.

\begin{tabular}{cccc}
\hline Threat & $t_{20}$ & $t_{35}$ & $t_{39}$ \\
\hline$T_{1}$ & 0.04 & 0.91 & 0.99 \\
$T_{2}$ & 0.05 & 0.94 & 1 \\
$T_{3}$ & 0.91 & 0.96 & 0.76 \\
$T_{4}$ & 0.92 & 0.95 & 0.74 \\
$T_{5}$ & 0.06 & 1 & 0.5 \\
\hline
\end{tabular}

Table 3: Normalised threat values associated with threats $T_{1}, \ldots, T_{5}$ during stages $t_{20}, t_{35}$ and $t_{39}$.

The efficiency values of the WSs with respect to the threats are all contained in an EEM, for each stage. The EEM for stage $t_{\alpha}$ is denoted by EEM $t_{t_{\alpha}}$. Terrain features and weather conditions are ignored for the sake of simplicity in the simulated scenario, and hence the EEMs contain only the SSHP information of WSs at the appropriate distances from the threats. The EEM instances $\mathrm{EEM}_{t_{20}}, \mathrm{EEM}_{t_{35}}$ and $\mathrm{EEM}_{t_{39}}$ are shown in Table 4 . Note, however, that the bi-objective WA model (1)-(4) requires the survival probabilities of the threats instead of the SSHP values of the WSs. The survival probability of a threat is the difference between unity and the corresponding SSHP value (i.e. the complement of the SSHP value).

Finally, the bi-objective WA model requires the cost of assigning a WS to a threat, which is taken here as the cost of a single burst of ammunition of a WS. It is assumed that the 


\begin{tabular}{|c|c|c|c|c|c|c|c|c|c|c|c|c|c|c|c|}
\hline \multicolumn{16}{|c|}{ Threats } \\
\hline$W S$ & $T_{1}$ & $T_{2}$ & $T_{3}$ & $T_{4}$ & $T_{5}$ & $T_{1}$ & $T_{2}$ & $T_{3}$ & $T_{4}$ & $T_{5}$ & $T_{1}$ & $T_{2}$ & $T_{3}$ & $T_{4}$ & $T_{5}$ \\
\hline$V_{1}$ & 0 & 0 & 0 & 0 & 0 & 0 & 0 & 0.1 & 0.5 & 0 & 0 & 0 & 0.1 & 0.1 & 0 \\
\hline$V_{2}$ & 0 & 0 & 0 & 0 & 0 & 0 & 0 & 0.3 & 0.1 & 0 & 0.7 & 0 & 0.5 & 0.5 & 0 \\
\hline$V_{3}$ & 0 & 0 & 0 & 0 & 0 & 0.1 & 0.5 & 0 & 0 & 0 & 0.1 & 0 & 0 & 0 & 0 \\
\hline$V_{4}$ & 0 & 0 & 0 & 0 & 0 & 0.1 & 0.5 & 0 & 0 & 0 & 0 & 0 & 0 & 0 & 0 \\
\hline$V_{5}$ & 0 & 0 & 0 & 0.5 & 0 & 0 & 0 & 0 & 0 & 0 & 0 & 0 & 0 & 0 & 0 \\
\hline$V_{6}$ & 0 & 0 & 0.1 & 0.1 & 0 & 0 & 0 & 0 & 0 & 0 & 0 & 0 & 0 & 0 & 0.1 \\
\hline$V_{7}$ & 0 & 0 & 0 & 0 & 0 & 0 & 0 & 0 & 0 & 0 & 0 & 0 & 0 & 0 & 0.5 \\
\hline$V_{8}$ & 0 & 0 & 0 & 0 & 0 & 0 & 0 & 0 & 0 & 0 & 0 & 0 & 0 & 0 & 0 \\
\hline$C_{1}$ & 0 & 0 & 0 & 0 & 0 & 0 & 0 & 0 & 0 & 0 & 0.2 & 0.4 & 0 & 0 & 0 \\
\hline$C_{2}$ & 0 & 0 & 0 & 0 & 0 & 0.2 & 0.3 & 0 & 0 & 0 & 0.5 & 0.9 & 0 & 0 & 0 \\
\hline$C_{3}$ & 0 & 0 & 0.1 & 0.1 & 0 & 0 & 0.1 & 0 & 0 & 0 & 0 & 0 & 0 & 0 & 0 \\
\hline$C_{4}$ & 0 & 0 & 0 & 0 & 0 & 0 & 0 & 0 & 0 & 0 & 0 & 0 & 0 & 0 & 0 \\
\hline
\end{tabular}

Table 4: The EEMs for stages $t_{20}, t_{35}$ and $t_{39}$.

cost of a single burst of ammunition for a VSHORAD WS is approximately R 1000000 , while the cost of a CIWS is approximately R 34000 . These costs were realistic in 2011.

\subsection{Implementation of the NSGA II}

The NSGA II requires a number of pre-specified parameter values. These values include the number of iterations over which the algorithm is applied, the size of the population of solutions, the tour size and pool size in the selection procedure, and the probability of a mutation occurring. In order to find a good spread of solutions along the approximately Pareto optimal front, these parameter values should be chosen carefully.

The number of iterations and size of the population were initially chosen randomly. The tour size involved in the selection process was chosen to be small, since a commonly employed value is between 2 and 5 . The pool size was chosen to be half the size of the population, since this is a typical choice of the pool size parameter in the selection procedure [11]. Furthermore, the probability of a mutation occurring should typically be relatively small. The commonly employed value of $1 / n^{*}$ was therefore adopted, where $n^{*}$ denotes the number of decision variables.

A sensitivity analysis was performed on this initial set of parameter values to find the best spread of Pareto optimal solutions. This was achieved by solving the WA problem for stage $t_{35}$ of the scenario using the initial parameter values and varying the values of each of these parameters separately while keeping the remaining parameter values fixed. The sensitivity analysis revealed that a good choice for the number of iterations is 350 and that a suitable choice for the population size is 200 . The tour and pool sizes in the selection process were therefore chosen to be 2 and 100, respectively - these values yielded the best spread of approximately Pareto optimal solutions. 


\subsection{Results obtained by means of the NSGA II}

As explained in $§ 4.3$, the NSGA II provides a number of solutions simultaneously in the bi-objective solution space. Since we are interested in the nondominated solutions, which are contained in the first front (i.e. the approximately Pareto optimal solutions), results are only presented in this section for solutions contained in the first front.

\subsubsection{The three approximately Pareto fronts for $t_{20}, t_{35}$ and $t_{39}$}

Stage $t_{20}$ yielded only five solutions along the approximate Pareto front. These solutions, together with their corresponding objective function values (accumulated survival probability and the cost in South African Rands (R)) as well as the proposed assignments of WSs to threats, are listed in Table 5 and presented graphically in Figure 4. The solutions corresponding to stage $t_{20}$ are represented by the black dots in the figure and are labelled $1,2, \ldots, 5$. In all five solutions threats $T_{1}, T_{2}$ and $T_{5}$ are not assigned any WSs, since all available WSs are only able to achieve SSHP values of zero with respect to these threats.

Solution 1 involves the largest number of WSs assigned (3), namely WSs $V_{6}$ and $C_{3}$ to threat $T_{3}$ and WS $V_{5}$ to threat $T_{4}$, achieving an accumulated survival probability of 1.3444 at an assignment cost of R 2034000 . Solution 5, on the other hand, involves no assignment of WSs to threats; the cost of the assignment is therefore zero. If no assignment of WSs is made, the accumulated survival probabilities of the five threats is 1.9754.

\begin{tabular}{clcr}
\hline Sol. & Assignment & $\begin{array}{c}\text { Accumulated } \\
\text { survival } \\
\text { probabilities }\end{array}$ & $\begin{array}{r}\text { Cost in } \\
\text { Rands }\end{array}$ \\
\hline 1 & $V_{6}, C_{3} \rightarrow T_{3} ; V_{5} \rightarrow T_{4} ;$ None $\rightarrow T_{1}, T_{2}, T_{5}$ & 1.3444 & $\mathrm{R} 2034000$ \\
2 & $C_{3} \rightarrow T_{3} ; V_{5} \rightarrow T_{4} ;$ None $\rightarrow T_{1}, T_{2}, T_{5}$ & 1.4260 & $\mathrm{R} 1034000$ \\
3 & $V_{5} \rightarrow T_{4} ;$ None $\rightarrow T_{1}, T_{2}, T_{3}, T_{5}$ & 1.5166 & $\mathrm{R} 1000000$ \\
4 & $C_{3} \rightarrow T_{4} ;$ None $\rightarrow T_{1}, T_{2}, T_{3}, T_{5}$ & 1.8836 & $\mathrm{R} 34000$ \\
5 & None $\rightarrow T_{1}, T_{2}, T_{3}, T_{4}, T_{5}$ & 1.9754 & $\mathrm{R} 0$ \\
\hline
\end{tabular}

Table 5: The set of approximately Pareto optimal solutions obtained by the NSGA II for stage $t_{20}$ of the scenario, using the same solution indices as in Figure 4.

Stage $t_{35}$ yielded fifteen solutions along the approximate Pareto front. The set of approximately Pareto optimal solutions, together with their corresponding objective function values as well as the proposed assignments of WSs to threats, are listed in Table 6 and are also presented graphically in Figure 4 . The solutions corresponding to stage $t_{35}$ are represented by the grey dots in the figure and are labelled $16,17, \ldots, 30$. Solution 16 involves the largest number of WSs (6) to be assigned during stage $t_{35}$, achieving an accumulated survival probability of 3.0925 at an assignment cost of R 4068000 , which is the largest cost solution for stage $t_{35}$, but achieves the lowest accumulated survival probability. Following the solutions from Solution 16 along the approximate Pareto optimal front in Figure 4, results in fewer proposed assignments yielding a decrease in the overall assignment cost and an increase in the accumulated survival probabilities of the threats up to Solution 30, where no assignments are proposed.

Finally, stage $t_{39}$ yielded ten solutions along the approximate Pareto front. These solu- 


\begin{tabular}{clcr}
\hline & & $\begin{array}{c}\text { Accumulated } \\
\text { survival } \\
\text { probabilities }\end{array}$ & $\begin{array}{r}\text { Cost in } \\
\text { Rands }\end{array}$ \\
Sol. & Assignment & 3.0925 & $\mathrm{R} 4068000$ \\
\hline 16 & $C_{2} \rightarrow T_{1} ; V_{3}, V_{4}, C_{3} \rightarrow T_{2} ; V_{2} \rightarrow T_{3} ; V_{1} \rightarrow T_{4} ;$ None $\rightarrow T_{5}$ & 3.1161 & $\mathrm{R} 4034000$ \\
17 & $C_{2} \rightarrow T_{1} ; V_{3}, V_{4} \rightarrow T_{2} ; V_{2} \rightarrow T_{3} ; V_{1} \rightarrow T_{4} ;$ None $\rightarrow T_{5}$ & 3.2991 & $\mathrm{R} 4000000$ \\
18 & $V_{3}, V_{4} \rightarrow T_{2} ; V_{2} \rightarrow T_{3} ; V_{1} \rightarrow T_{4} ;$ None $\rightarrow T_{1}, T_{5}$ & 3.3050 & $\mathrm{R} 3068000$ \\
19 & $C_{2} \rightarrow T_{1} ; V_{3}, C_{3} \rightarrow T_{2} ; V_{2} \rightarrow T_{3} ; V_{1} \rightarrow T_{4} ;$ None $\rightarrow T_{5}$ & 3.3522 & $\mathrm{R} 3034000$ \\
20 & $C_{2} \rightarrow T_{1} ; V_{3} \rightarrow T_{2} ; V_{2} \rightarrow T_{3} ; V_{1} \rightarrow T_{4} ;$ None $\rightarrow T_{5}$ & 3.5351 & $\mathrm{R} 3000000$ \\
21 & $V_{3} \rightarrow T_{2} ; V_{2} \rightarrow T_{3} ; V_{1} \rightarrow T_{4} ;$ None $\rightarrow T_{1}, T_{5}$ & 3.5939 & $\mathrm{R} 2068000$ \\
22 & $C_{2} \rightarrow T_{1} ; V_{3}, C_{3} \rightarrow T_{2} ; V_{1} \rightarrow T_{4} ;$ None $\rightarrow T_{3}, T_{5}$ & 3.6412 & $\mathrm{R} 2034000$ \\
23 & $C_{2} \rightarrow T_{1} ; V_{3} \rightarrow T_{2} ; V_{1} \rightarrow T_{4} ;$ None $\rightarrow T_{3}, T_{5}$ & 3.8240 & $\mathrm{R} 2000000$ \\
24 & $V_{3} \rightarrow T_{2} ; V_{1} \rightarrow T_{4} ;$ None $\rightarrow T_{1}, T_{3}, T_{5}$ & 3.9468 & $\mathrm{R} 1068000$ \\
25 & $C_{2}, C_{3} \rightarrow T_{2} ; V_{1} \rightarrow T_{4} ;$ None $\rightarrow T_{1}, T_{3}, T_{5}$ & 4.0129 & $\mathrm{R} 1034000$ \\
26 & $C_{2} \rightarrow T_{2} ; V_{1} \rightarrow T_{4} ;$ None $\rightarrow T_{1}, T_{3}, T_{5}$ & 4.2962 & $\mathrm{R} 1000000$ \\
27 & $V_{1} \rightarrow T_{4} ;$ None $\rightarrow T_{1}, T_{2}, T_{3}, T_{5}$ & 4.4209 & $\mathrm{R} 68000$ \\
28 & $C_{2}, C_{3} \rightarrow T_{2} ;$ None $\rightarrow T_{1}, T_{3}, T_{4}, T_{5}$ & 4.4870 & $\mathrm{R} 34000$ \\
29 & $C_{2} \rightarrow T_{2} ;$ None $\rightarrow T_{1}, T_{3}, T_{4}, T_{5}$ & 4.7703 & $\mathrm{R} 0$ \\
30 & None $\rightarrow T_{1}, T_{2}, T_{3}, T_{4}, T_{5}$ & & \\
\hline
\end{tabular}

Table 6: The set of approximately Pareto optimal solutions obtained by the NSGA II for stage $t_{35}$ of the scenario, using the same solution indices as in Figure 4.

tions, together with their corresponding objective function values as well as the proposed assignments of WSs to threats, are listed in Table 7 and are yet again presented graphically in Figure 4 . The solutions corresponding to stage $t_{39}$ are represented by black circled dots in the figure and are labelled $6,7, \ldots, 15$.

\subsubsection{Focusing on the results for stage $t_{39}$}

Solution 6 in the set of approximately Pareto optimal solutions for stage $t_{39}$ entails the worst possible value for the cost objective, achieving an accumulated survival probability of 1.9613 at an assignment cost of R 5068000 . Since WS $V_{2}$ achieves an SSHP value of 0.7 with respect to threat $T_{1}$, as opposed to an SSHP value of 0.5 with respect to threat

\begin{tabular}{clcr}
\hline & & $\begin{array}{c}\text { Accumulated } \\
\text { survival } \\
\text { probabilities }\end{array}$ & $\begin{array}{r}\text { Cost in } \\
\text { Rands }\end{array}$ \\
\hline 6 & Assignment & 1.9613 & $\mathrm{R} 5068000$ \\
7 & $V_{2}, V_{3}, C_{1} \rightarrow T_{1} ; C_{2} \rightarrow T_{2} ; V_{1} \rightarrow T_{3} ; V_{6}, V_{2} \rightarrow V_{2} ; V_{1} \rightarrow T_{5} ; V_{6}, V_{7} \rightarrow T_{5} ;$ None $\rightarrow T_{4} \rightarrow T_{4}$ & 1.9852 & $\mathrm{R} 4068000$ \\
8 & $V_{2}, C_{1} \rightarrow T_{1} ; C_{2} \rightarrow T_{2} ; V_{1} \rightarrow T_{3} ; V_{7} \rightarrow T_{5} ;$ None $\rightarrow T_{4}$ & 2.0102 & $\mathrm{R} 3068000$ \\
9 & $V_{2} \rightarrow T_{1} ; C_{2} \rightarrow T_{2} ; V_{1} \rightarrow T_{3} ; V_{7} \rightarrow T_{5} ;$ None $\rightarrow T_{4}$ & 2.0702 & $\mathrm{R} 3034000$ \\
10 & $V_{2}, C_{1} \rightarrow T_{1} ; C_{2} \rightarrow T_{2} ; V_{7} \rightarrow T_{5} ;$ None $\rightarrow T_{3}, T_{4}$ & 2.0858 & $\mathrm{R} 2068000$ \\
11 & $V_{2} \rightarrow T_{1} ; C_{2} \rightarrow T_{2} ; V_{7} \rightarrow T_{5} ;$ None $\rightarrow T_{3}, T_{4}$ & 2.1457 & $\mathrm{R} 2034000$ \\
12 & $V_{2}, C_{1} \rightarrow T_{1} ; C_{2} \rightarrow T_{2} ;$ None $\rightarrow T_{3}, T_{4}, T_{5}$ & 2.3358 & $\mathrm{R} 1068000$ \\
13 & $V_{2} \rightarrow T_{1} ; C_{2} \rightarrow T_{2} ;$ None $\rightarrow T_{3}, T_{4}, T_{5}$ & 2.3957 & $\mathrm{R} 1034000$ \\
14 & $C_{1} \rightarrow T_{1} ; C_{2} \rightarrow T_{2} ;$ None $\rightarrow T_{3}, T_{4}, T_{5}$ & 2.8952 & $\mathrm{R} 68000$ \\
15 & $C_{2} \rightarrow T_{2} ;$ None $\rightarrow T_{1}, T_{3}, T_{4}, T_{5}$ & 3.0949 & $\mathrm{R} 34000$ \\
\hline
\end{tabular}

Table 7: The set of approximately Pareto optimal solutions obtained by the NSGA II for stage $t_{39}$ of the scenario, using the same solution indices as in Figure 4. 


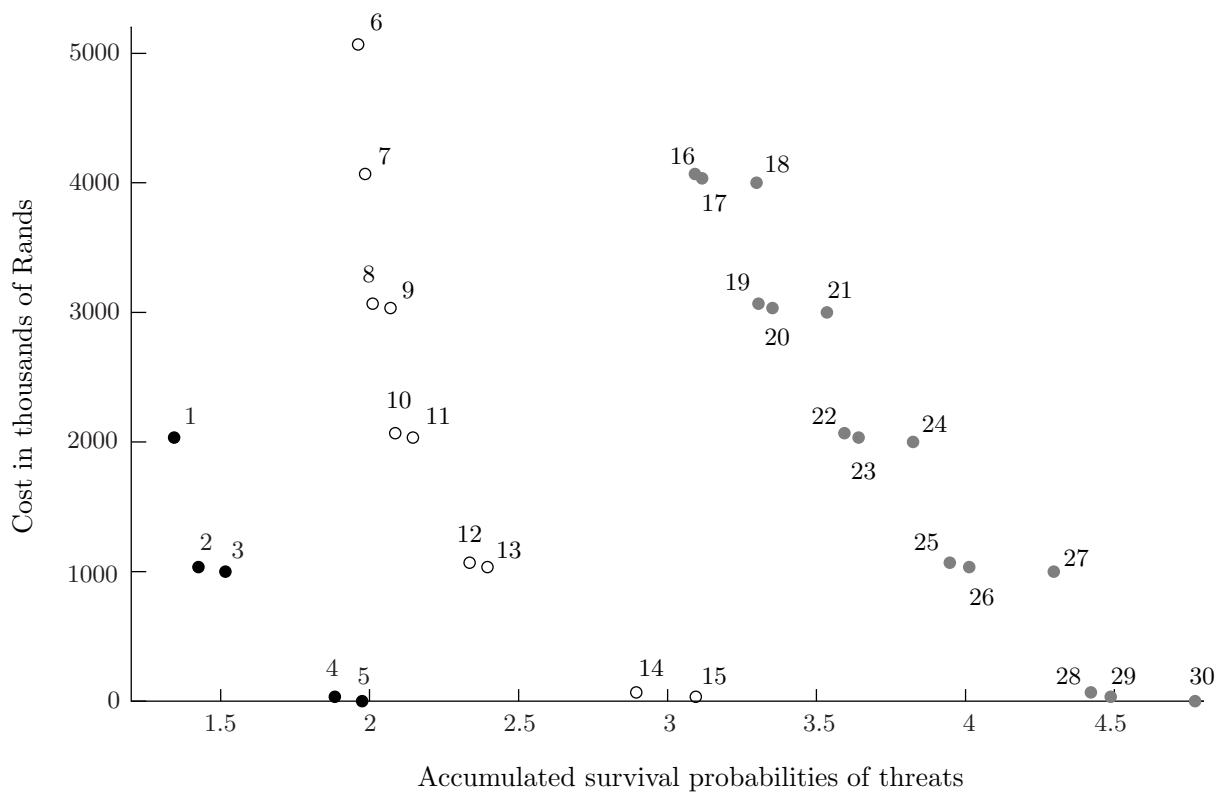

Figure 4: Graphical illustration of the sets of approximately Pareto optimal solutions obtained by means of the NSGA II for stages $t_{20}$ (solutions 1-5), $t_{39}$ (solutions $6-15$ ) and $t_{35}$ (solutions 16-30) of the scenario in $\S 5.1$.

$T_{4}$, together with the fact that threat $T_{1}$ is considered a higher priority threat than threat $T_{4}$, WS $V_{2}$ is assigned to threat $T_{1}$ rather than being assigned to threat $T_{4}$. The same reason applies for assigning WS $V_{1}$ to threat $T_{3}$ rather than assigning it to threat $T_{4}$. The remaining WSs achieve an SSHP of zero with respect to threat $T_{4}$. Hence, no WS is assigned to threat $T_{4}$. The assignments of WSs to threats for Solution 6 (stage $t_{39}$ ) are presented graphically in Figure 5(a).

Analysing the solutions along the front of approximately Pareto optimal solutions for stage $t_{39}$ in Figure 4, consider, for example, Solution 11 which achieves an accumulated survival probability of 2.1457 at an assignment cost of R 2034000 . The assignments contained in this solution are similar to the assignments proposed in Solution 6, except for the assignment of only WS $V_{2}$ to threat $T_{1}$, rather than assigning WSs $V_{2}, V_{3}$ and $C_{1}$ to threat $T_{1}$, while no assignments are made to threat $T_{3}$ rather than assigning WS $V_{1}$ to threat $T_{3}$. The result of this assignment is a decrease in the cost value of $\mathrm{R} 3034000$ and an increase in the accumulated survival probabilities of threats of 0.1844 . The proposed assignments corresponding to Solution 11 for stage $t_{39}$ are presented graphically in Figure $5(\mathrm{~b})$.

Finally, consider Solution 15 on the front of approximately Pareto optimal solutions for stage $t_{39}$ in Figure 4, achieving an accumulated survival probability of 3.0949 at an assignment cost of R 34000 . This solution involves the best possible value for the cost objective and achieves the worst possible value for the accumulated survival probability objective at stage $t_{39}$. Similar assignments are proposed as in Solution 11, except that no WSs are assigned to threat $T_{1}$ rather than assigning WS $V_{2}$ to threat $T_{1}$ and no WSs are assigned to threat $T_{5}$ rather than assigning WS $V_{7}$ to threat $T_{5}$. This results in a further decrease in the total cost value of R 2000000 and an increase in the total survival probabilities of 


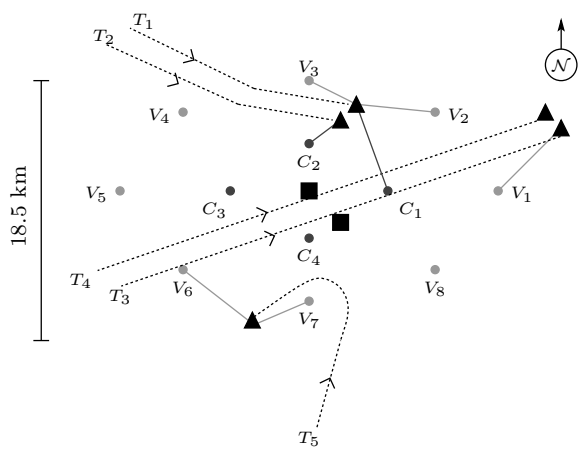

(a) Assignments for solution 6

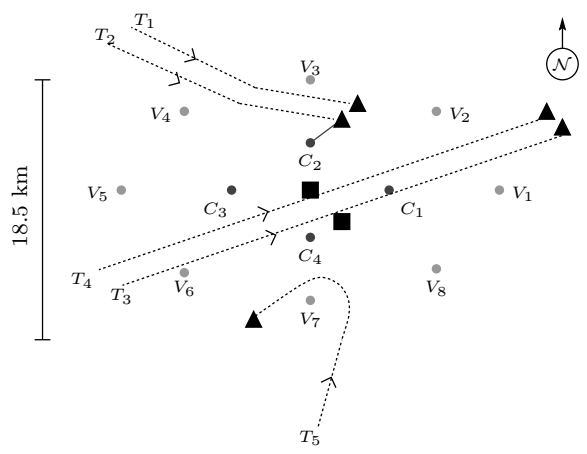

(c) Assignments for solution 15

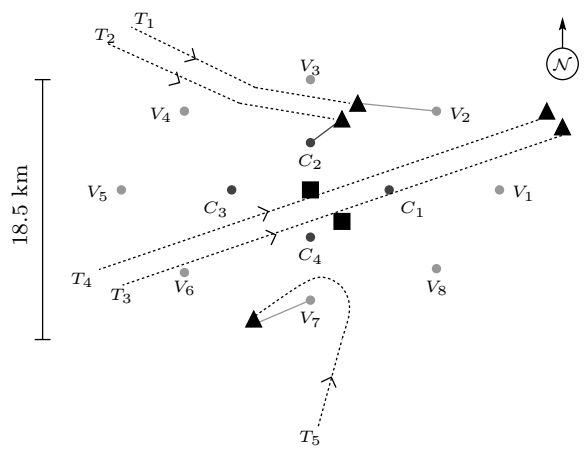

(b) Assignments for solution 11

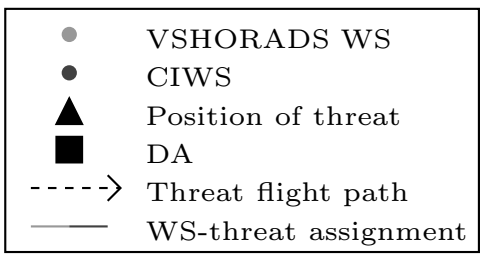

(d) Legend

Figure 5: Top-view graphical illustration of the assignment of WSs to threats obtained by means of the NSGA II for Solutions 6, 11 and 15, respectively, at stage $t_{39}$.

threats of 0.9492 . The proposed assignments of WSs to threats in Solution 15 for stage $t_{39}$ are presented graphically in Figure $5(\mathrm{c})$. In contrast to stages $t_{20}$ and $t_{35}$, the cost function of the final solution is not zero. This may be due to the fact that threat $T_{2}$ involves a threat value of one (the maximum threat value that may possibly be assigned to a threat) and the fact that WS $C_{2}$ achieves an SSHP value of 0.9 with respect to threat $T_{2}$, if WS $C_{2}$ is assigned to threat $T_{2}$.

\subsection{Advantages of using a population-based solution approach}

An advantage of a population-based solution approach, such as the NSGA II, is that it is able to provide the decision maker with a set of approximately Pareto optimal solutions of assignments. The approximately Pareto optimal solutions provide the decision maker with a useful trade-off between the objectives, illustrating how much of one objective is offered for a gain in the other objective.

Another advantage is that the objective functions may also be constrained, individually or together, after which part of the approximately Pareto optimal set of solutions may still be recommended to the decision maker. In the context of the scenario, suppose the decision maker constrains the cost objective by indicating that the maximum allowable 


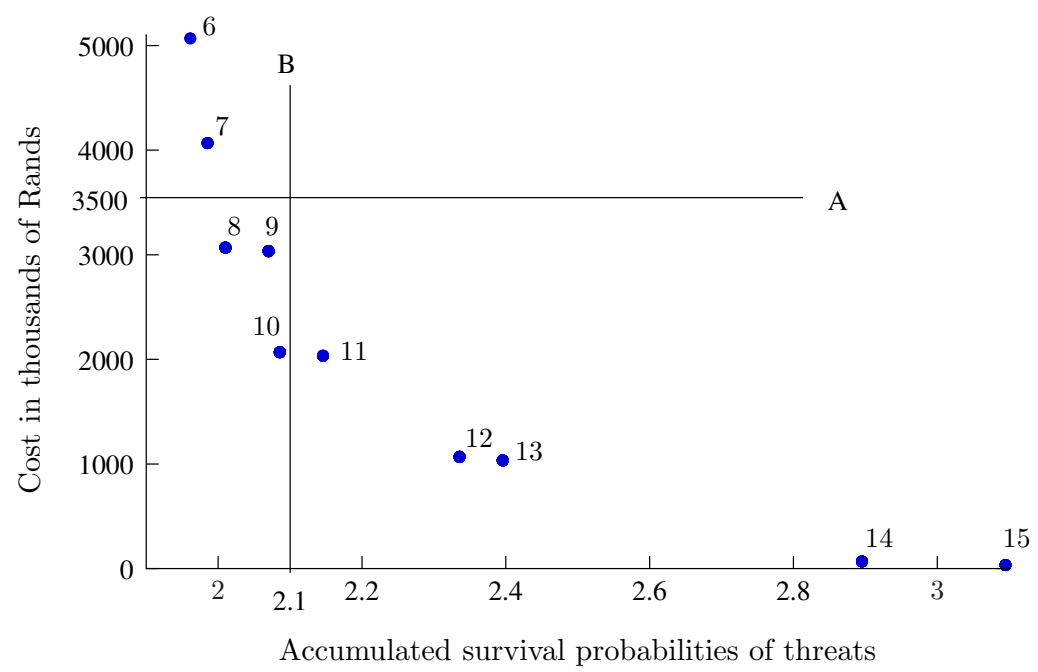

Figure 6: Graphical illustration of the set of approximately Pareto optimal solutions obtained by means of the NSGA II for stage $t_{39}$ of the scenario, when the bi-objective WA problem is constrained.

cost of assigning WSs to threats is R 3500000 . This constraint is presented graphically by the horizontal line labelled $\mathrm{A}$ in Figure 6 and implies that no solution above this line (i.e. Solutions 6 and 7) should be recommended. Any of the solutions below this line may, however, be recommended as a possible assignment of WSs to threats. The accumulated survival probability objective may be constrained similarly. Suppose, alternatively, that a maximum acceptable value for the accumulated survival probability is 2.1 . This constraint is presented graphically by the vertical line labelled B in Figure 6 and implies that any solution to the left of the line B may be considered as allowable assignments of WSs to threats, while Solutions 11-15 may not be considered. The possibility also exists that both objectives may be constrained. Suppose that the value of cost is restricted to at most R 3500000 and that the accumulated survival probability of the threats is restricted to at most 2.1. This implies that any solution below the line A and to the left of the line B may be considered for a possible assignment of WSs to threats. Hence, only Solutions 8, 9 and 10 may be considered.

Finally, the NSGA II is flexible in the sense that it is able to accommodate a variation in the input values of the objectives which fall outside the range of the current values employed. Consider the scenario in $§ 5.1$. If, for example, a WS were to be added to the current scenario described in $§ 5.1$ which involves an ammunition cost of R 6000000 , the NSGA II can easily be adjusted to accommodate such a WS.

\section{Future work}

Although the results presented in this paper seem plausible, three future developments are discussed in this section which may lead to more sophisticated multiobjective WA models. A first suggestion is to identify suitable WA objectives by means of a more comprehensive 
approach. An interactive workshop in the presence of a workshop facilitator in conjunction with a number of military experts, spanning a considerable portion of the hierarchical ranks contained in the South African military domain, may lead to a more comprehensive set of fundamental objectives. In this way the input from a diverse set of individuals may be obtained in an environment where the military experts are free to contribute ideas anonymously and thus to question the views of their peers or superiors. The result of hosting such a workshop may lead to a consensus with respect to the objectives related to the multiobjective WA problem in a South African GBAD context.

The multiobjective WA model in this paper should also be solved in the context of other simulated GBAD scenarios, rather than just solving it in the context of one scenario.

Finally, the bi-objective model devised in this paper considered only a single stage at a time. The possibility also exists to model the bi-objective WA model for the current period as well as a number of future stages, thereby introducing a dynamic, scheduling element into the static WA model. Time windows may then be included in the model, during which a WS is available for assignment to a threat. Van der Merwe [19] investigated the possibility of formulating and solving a single-objective WA model at the current stage as well as for a number of future stages. Her work may be used in conjunction with the multiobjective WA model presented in this paper as a point of departure to formulate a more realistic mulitobjective, multi-stage WA model.

\section{References}

[1] Agarwal S, Deb K, Meyarivan T \& Pratap A, 2002, A fast and elitist multiobjective genetic algorithm: NSGA-II, IEEE Transactions on Evolutionary Computation, 6(2), pp. 182-197.

[2] Ahuja RK, Kumar A, Jha KC \& Orlin JB, 2003, Exact and heuristic algorithms for the weapon target assignment problem, MIT Sloan, Working Paper No. 4464-03.

[3] Belton V \& Stewart TJ, 2002, Multiple criteria decision analysis: An integrated approach, Kluwer Academic Publishers, Boston (MA).

[4] Coello Coello CA, Lamont GB \& Van Velduizen DA, 2007, Evolutionary algorithms for solving multi-objective problems, $2^{\text {nd }}$ Edition, Springer Science, New York (NY).

[5] Deb K \& SRinivas N, 1995, Multiobjective optimisation using nondominated sorting genetic algorithms, Journal of Evolutionary Computation, 2(3), pp. 221-248.

[6] Farlex, 2011, The free dictionary: Post it, [Online], [Cited October 18 ${ }^{\text {th }}$, 2011], Available from http://encyclopedia. thefreedictionary. com/Post+it

[7] Flood M, 1957, Target-assignment model, Proceedings of the Princeton University Conference on Linear Programming, Princeton (NJ).

[8] Hartman FT, Krahn J \& Skulmoski GJ, 2007, The Delphi method for graduate research, Journal of Information Technology Education, 6(1), pp. 1-21.

[9] KeEney RL, 1996, Value-focused thinking: Identifying decision opportunities and creating alternatives, European Journal of Operational Research, 92(3), pp. 537-549.

[10] Linstone HA \& Turoff M, 1975, The Delphi method: Techniques and applications, Addison-Wesley Publishing Company, Reading (MA).

[11] Mathworks: MATlaB Central, 2009, NSGA II: A multiobjective opimisation algorithm by Aravind Seshandri, [Online], [Cited November $6^{\text {th }}, 2011$ ], Available from http://www.mathworks.com/

[12] Manne AS, 1958, A target-assignment problem, Operations Research, 6(3), pp. 346-351.

[13] Potgieter JJ, 2008, Real-Time weapon assignment in a ground-based air defence environment, MSc Thesis, Stellenbosch University, Stellenbosch. 
[14] Potgieter JJ, 2010, Software Engineer: Reutech Radar Systems, [Personal Communication], Contactable at cobusp@reutech.co.za.

[15] Roux JN, 2010, Project Engineer: Reutech Radar Systems, [Personal Communication], Contactable at jacor@reutech.co.za.

[16] Roux JN, 2010, Design of a threat evaluation subsystem in a ground-based air defence environment, PhD Dissertation, Stellenbosch University, Stellenbosch.

[17] Roux JN \& VAN VuURen JH, 2007, Threat evaluation and weapon assignment decision support: A review of the state of the art, ORiON, 23(2), pp. 151-187.

[18] Roux JN \& VAn Vuuren JH, 2008, Real-time threat evaluation in a ground-based air defence environment, ORiON, 24(1), pp. 75-101.

[19] Van Der Merwe M, 2010, Masters Student: Department of Logistics, Stellenbosch University, [Personal Communication], Contactable at michelle@wigroup.co.za.

[20] Visser B, 2011, Military Expert: Reutech Radar Systems, [Personal Communication], Contactable at bvisser@rrs.co.za.

[21] Votaw DF \& Orden A, 1952, The personnel assignment problem, Symposium on Linear Inequalities and Programming, SCOOP 10(1), US Air Force, pp. 155-163.

\section{Appendix: Questionnaire circulated to military experts}

For the purpose of this questionnaire, suppose that all the scenarios are contained within a typical GBAD environment, where DAs have to be protected against incoming aerial threats. Each set of questions is based on a specific scenario subject to different conditions. Please note that these scenarios are simplified versions of real-world scenarios. Consider only the given conditions and please complete the questions from the perspective of a TEWA system ${ }^{5}$.

The following conditions apply throughout the entire questionnaire, unless explicitly stated otherwise:

1. All detected threats are considered fixed wing aircraft.

2. Weather conditions are considered to be daytime with sunny skies and no wind.

3. The dotted lines in each scenario represent the flight paths of threats.

4. Each scenario involves one DA represented by a large grey dot.

5. Each detected threat is travelling at a speed of $250 \mathrm{~m} / \mathrm{s}$.

6. In each scenario one of each of the following types of WSs, represented by squares, is available:

(a) $35 \mathrm{~mm}$ cannon section,

(b) Starstreak system,

(c) Umkhonto system.

7. All WSs are deployed and "ready for action."

8. The threat positions A and B (represented by small black dots) in the scenarios are located at various distances from the WSs.

9. A maximum of one WS may be considered for assignment with respect to a threat at each position, i.e. either one of the WSs may be selected or none at all, in which case it is implied that the threat should be engaged at a later stage.

10. Note that threat positions $\mathrm{A}$ and $\mathrm{B}$ are independent of each other, i.e. when WSs are considered for assignment to a threat at position $\mathrm{B}$, the proposed assignment at position $\mathrm{A}$ may be ignored.

11. In each of the scenarios, threat position A is located $12 \mathrm{~km}$ from the WSs and threat position B is located $8 \mathrm{~km}$ from the WSs, which is on the OP warning line.

\footnotetext{
${ }^{5}$ Each question in the questionnaire consisted of two parts. The first part required the military expert to choose a WS based on a specific scenario and the second part required the military expert to state a reason for his answer. However, the second part of each question is omitted here due to space restrictions.
} 


\section{Scenario 1}

1.1. Which WS would you like a TEWA DSS to propose for engaging the threat at the indicated positions A and B of Figure 7, respectively?

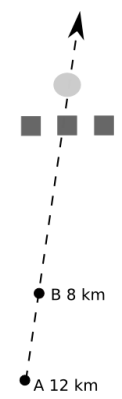

Figure 7: Scenario 1 for survey questions 1.1-1.6 and 4.1-4.8.

1.2. If the weather conditions change to heavy rainfall, which WS would you like a TEWA DSS to propose for engaging the threat at the indicated positions A and B, respectively?

1.3. Suppose that it is sunny weather conditions, but with a very strong cross wind $(>60 \mathrm{~km} / \mathrm{h}$ ). Which WS would you like a TEWA DSS to propose for engaging the threat at positions $\mathrm{A}$ and $\mathrm{B}$, respectively?

1.4. Now suppose it is night time, with clear skies and no wind. Which WS would you like a TEWA DSS to propose for engaging the threat at the indicated positions A and B, respectively?

1.5. If the weather is sunny with no wind and the threat is travelling at $150 \mathrm{~m} / \mathrm{s}$, which WS would you like a TEWA DSS to propose for engaging the threat at positions A and $\mathrm{B}$, respectively?

1.6. If the weather is sunny with no wind and the threat is travelling at $300 \mathrm{~m} / \mathrm{s}$, which WS would you like a TEWA DSS to propose for engaging the threat at positions A and $\mathrm{B}$, respectively?

\section{Scenario 2}

2.1. Suppose that a WS may only be assigned to exactly one threat at a time. Which WS would you like a TEWA DSS to assign for engaging the threats at positions A1, A2, B1 and B2 of Figure 8(a), respectively?

2.2. Suppose threat 1 is travelling at $150 \mathrm{~m} / \mathrm{s}$, while threat 2 travels at $250 \mathrm{~m} / \mathrm{s}$. Which WS would you like a TEWA DSS to assign to the threats at positions A1, A2, B1 and B2, respectively?

2.3. Suppose threat 1 is only detected at position B1 and travelling at $250 \mathrm{~m} / \mathrm{s}$, while threat 2 is still detected at position A2. Which WS would you like a TEWA DSS to assign to the threats at positions $\mathrm{A} 2, \mathrm{~B} 1$ and $\mathrm{B} 2$, respectively? 


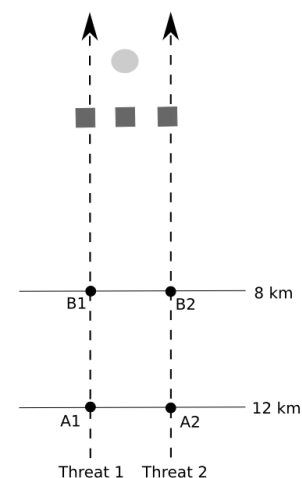

(a)

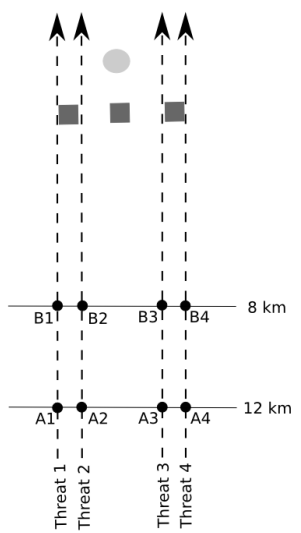

(b)

Figure 8: Scenario 2 for (a) survey questions 2.1-2.4 and (b) survey question 2.5.

2.4. Suppose that a WS may be assigned to more than one threat at a time. Which WS would you like a TEWA DSS to assign to the threats at positions A1, A2, B1 and B2, respectively?

2.5. Now suppose that there are four detected threats as may be seen in Figure 8(b), and that a WS may be assigned to more than one threat at a time. Which WS would you like a TEWA DSS to assign to the threats at positions A1, A2, B1 and B2, respectively?

\section{Scenario 3}

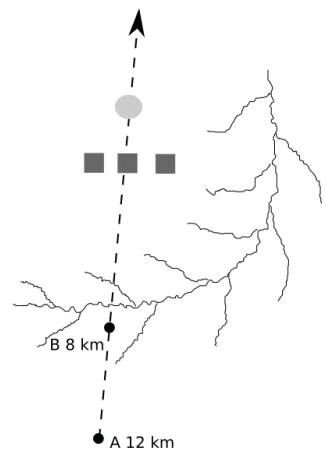

Figure 9: Scenario 3 for survey question 3.

3. In this scenario the WSs and DAs are partially surrounded by a mountain range, as indicated in Figure 9. Due to the mountain range, there is no line of sight between the WSs and the threat at position A; the threat only comes into line of sight at position $B$. However, it is detected by radar at position $A$. Which one of the three WSs would you like a TEWS DSS to assign for engaging the threat at positions A and B, respectively? 


\section{Scenario 4}

4.1. Consider the same situation as in Scenario 1. Additionally, take the monetary cost of one Umkhonto missile as R2 million, the monetary cost of one Starstreak missile as $R 1$ million and the monetary cost of a single burst of ammunition for a 35mm Cannon section as $R 17000$. Considering the monetary cost of assigning a WS to a threat, which one of these WS would you like a TEWA DSS to propose WA for engaging the threat at position B?

4.2. Are there any factors, other than the monetary cost of ammunition, which should be included in calculations by a TEWA DSS when assigning WSs to a threat?

4.3. Consider the monetary cost of assigning a Starstreak with a predicted SSHP of 0.9, and a $35 \mathrm{~mm}$ with a predicted SSHP of 0.7 at position $B$. Which one of these WS would you like a TEWA DSS to propose for engaging the threat at position B?

4.4. Under which circumstances would you like a TEWA DSS to rather propose a WS which is less capable (smaller SSHP), because it is cheaper to assign?

4.5. Suppose the Starstreak has one round of ammunition (hot; and no cold) available, and the $35 \mathrm{~mm}$ has 50 bursts of ammunition available. Would you like a TEWA DSS to propose the Starstreak or the $35 \mathrm{~mm}$ ? Why did you choose this WS?

4.6. Under which circumstances would you like TEWA to rather propose a WS with more ammunition than a WS with less ammunition.

4.7. Do you think that ammunition available to a WS should be considered a factor in your decision to assign a WS? Simply answer by stating only YES or NO.

5. Do you think the following factors should be considered when deciding which WSs to propose to threats? A simple YES or NO answer next to each is sufficient.

5.1. SSHP

5.2. Cost of ammunition

5.3. Available ammunition to a WS

5.4. Line of sight

5.5. Weather conditions

5.6. Vertical launch ability of a WS

5.7. Multiple engagements of a WS

5.8. Terrain

5.9. Reaction time of a WS

5.10. Speed of WS ammunition

5.11. Effective range of a WS

6. Can you think of any other factors not covered in this questionnaire that you feel should be included in the decision of assigning WSs to threats? 